\title{
Metastatic Meningioma of the Neck: A Case Report and Systematic Review
}

\author{
Hoang C.B. Nguyen ${ }^{a} \quad$ Leila J. Mady $^{b}$ Kush Panara $^{b}$ Stefen Andrianus ${ }^{c}$ \\ Kumarasen Cooper ${ }^{c}$ Isaac H. Chen ${ }^{d}$ Ara A. Chalian ${ }^{b}$ Robert M. Brody ${ }^{b}$ \\ aPerelman School of Medicine, University of Pennsylvania, Philadelphia, PA, USA; bepartment of \\ Otorhinolaryngology-Head and Neck Surgery, University of Pennsylvania, Philadelphia, PA, USA; \\ 'Department of Pathology and Laboratory Medicine, University of Pennsylvania, Philadelphia, PA, USA; \\ ${ }^{\mathrm{d}}$ Department of Neurosurgery, University of Pennsylvania, Philadelphia, PA, USA
}

\section{Keywords}

Metastatic meningioma $\cdot$ Cervical lymph node $\cdot$ Neck mass

\begin{abstract}
Background: Although meningiomas are the most common central nervous system neoplasms, extracranial metastases are exceedingly rare. There are even fewer reports of metastatic meningiomas to the neck. Methods: We described a patient with multiply recurrent orbital meningioma with metastasis to the neck found incidentally during neck exploration for composite resection and free tissue reconstruction. We performed a systematic review for all records pertaining to metastatic meningiomas to the cervical regions. Results: We found 9 previous reports of cervical metastatic meningiomas. Almost all cases underwent extensive local resection. There was no evidence of an association between the histological grade of the tumor and risk of metastasis to the neck. Cervical lymph node dissemination is more common in patients presenting after previous primary tumor resection. Conclusions: In the context of a neck mass, our findings suggest that metastatic meningioma should be included in the differential diagnosis, especially in patients with previous resections.

(c) 2022 S. Karger AG, Basel
\end{abstract}

\section{Introduction}

In the central nervous system (CNS), the meninges are tissue layers that serve to encapsulate both the brain and the spinal cord. Meningiomas which arise from these tissues most commonly originate from a subgroup of arachnoid cells known as meningothelial arachnoid cells, which are morphologically distinct and metabolically active. The embryological origins of these cell types are also location dependent and help to explain the different histological subtypes of meningiomas. Tumors that develop from the convexity of the brain are neural crest in origin, whereas those that originate from the skull base are mesoderm derivatives $[1,2]$.

Meningiomas are one of the most common CNS tumors, with an incidence of roughly 8 per 100,000 persons per year [3]. The majority of meningiomas are benign (grade I) per the 2007 WHO classification system. Meningiomas with more aggressive behavior, higher risk of local recurrence, and poorer prognosis are classified as atypical (grade II) or anaplastic (grade III), accounting for roughly $20 \%$ of cases [4]. Most meningiomas occur in the older population, with the majority aged 65 years and over. In adulthood, these tumors have a predilection for females, with a reported $3: 1$ female to male ratio $[3,5]$.

Correspondence to:

Robert M. Brody, robert.brody@pennmedicine.upenn.edu 


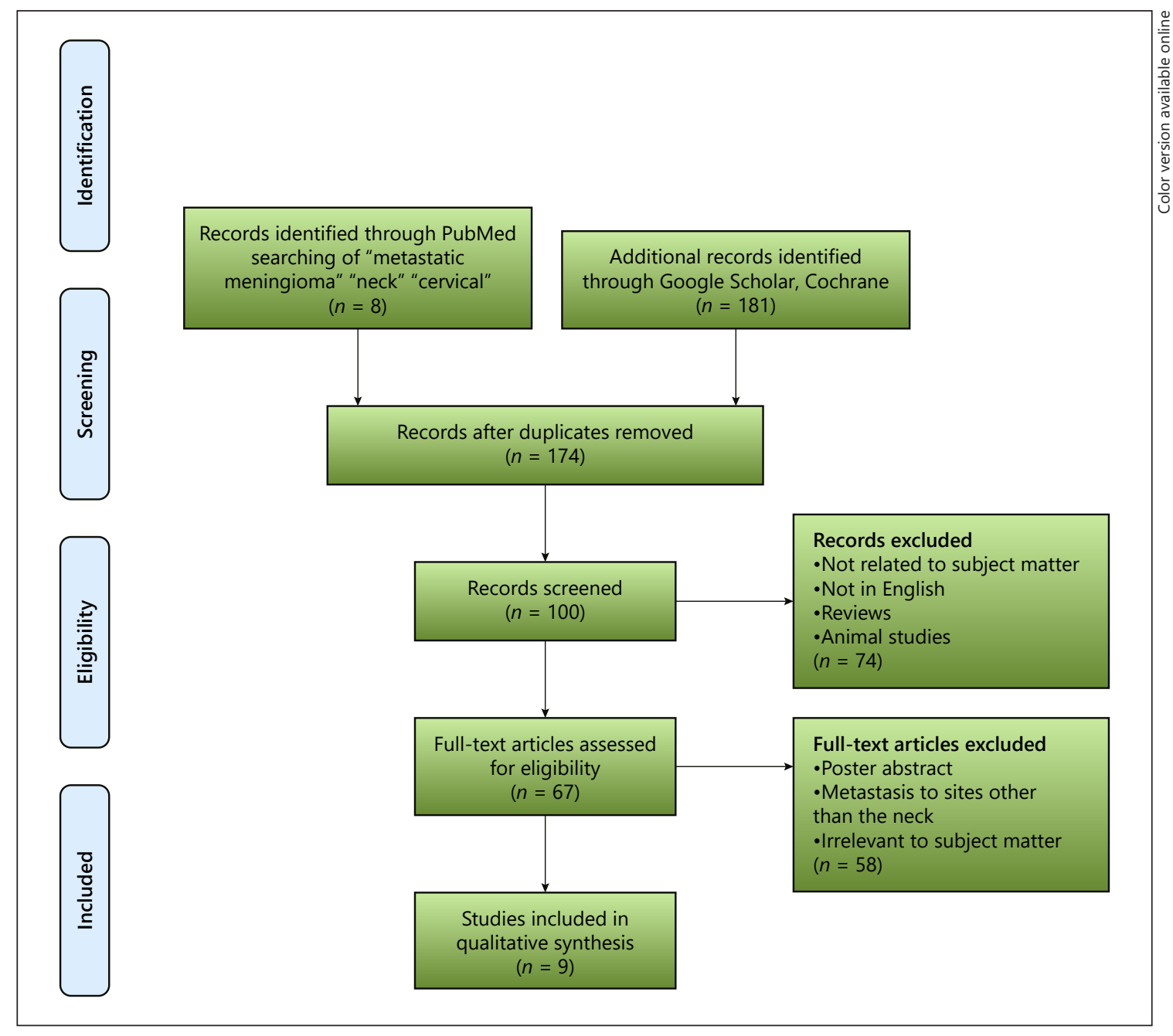

Fig. 1. Flow diagram of systematic literature review.

Childhood incidence of meningiomas are exceedingly uncommon, accounting for less than $5 \%$ of all pediatric tumors, with most cases associated with germline mutations in the neurofibromin 2 gene (NF2) [6]. As is common for pediatric tumors, meningiomas in children often exhibit a higher degree of malignancy than their adult counterparts $[7,8]$.

Metastatic meningiomas are exceedingly rare $(0.1 \%)$, and metastases can occur in nonmalignant subtypes [9]. There are numerous reports of grade I meningiomas with extracranial manifestations, most commonly in the lungs, liver, and bone [5, 10-14]. Less common, however, are reports of meningioma that are metastatic to the neck. Here, we report a rare case of orbital meningioma with metastasis to the cervical lymph node basin, as well as a descriptive systematic review of previously reported cases. We also highlight the most recent advances in molecular diagnostics of meningiomas. With recent developments in next-generation high-throughput sequencing, there is an emerging understanding of the genetic and epigenetics aberrations associated with recurrent and aggressive disease [15].

\section{Methods}

According to the University of Pennsylvania's Office of the Vice Provost for Research, a case report does not require approval from the institutional review board (IRB) as it did not involve subjects' research. In addition, all individuals involved in our case study and reviews were de-identified in compliance with HIPAA 
Fig. 2. Axial contrast-enhanced T1-weighted MRI images of the head preoperatively at the level of a the orbit showing a $25.4 \mathrm{~mm}$ $\times 2.0 \mathrm{~mm}$ enhancing mass in the left orbit and $\mathbf{b}$ zygomatic arch showing a $34.1 \mathrm{~mm}$ $\times 33.1 \mathrm{~mm}$ mass in the left masticator space centered around the coronoid process of the ascending ramus of the mandible, extending to involve the pterygoid and masseter muscles.
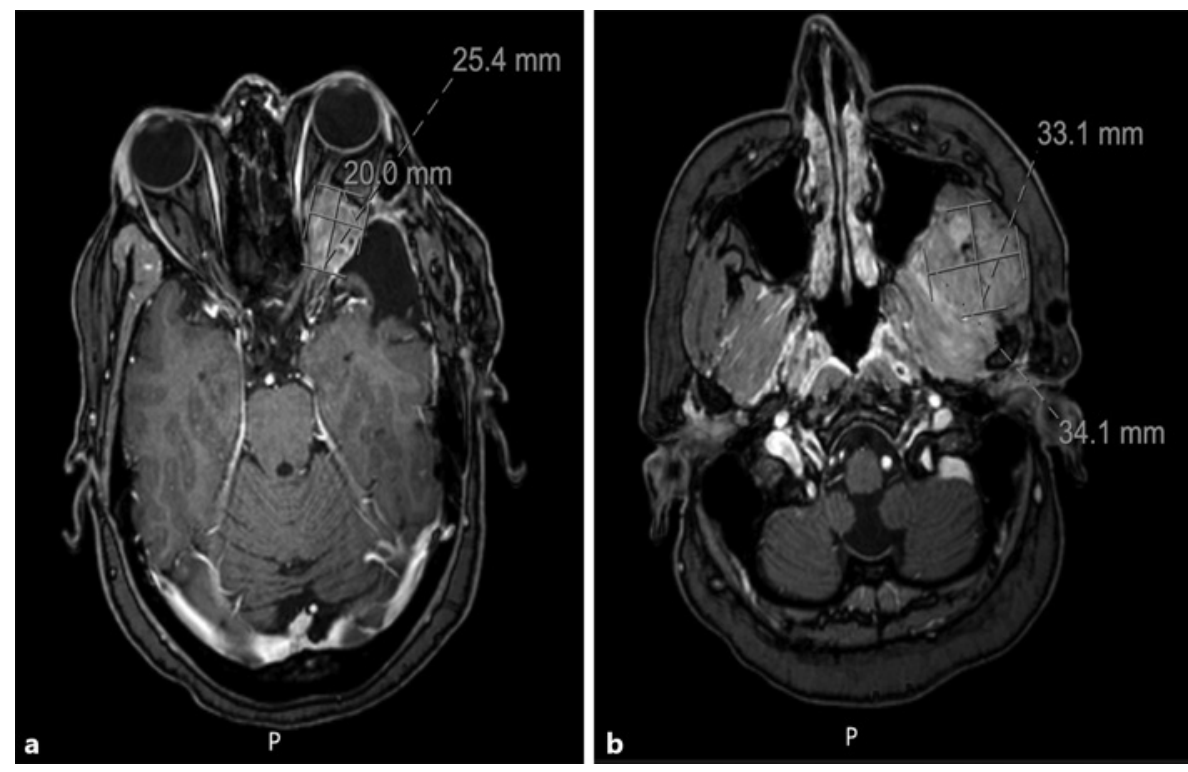

guidelines. As such, no informed consent was sought from the patients.

A systematic review was performed according to the guidelines of the Preferred Reporting Items for Systematic Reviews and MetaAnalyses (PRISMA) [16] (Fig. 1). A PRISMA Checklist is provided as an online supplementary File (for all online suppl. material, see www.karger.com/doi/10.1159/000521076). The review was conducted to answer the question: "Has cervical metastasis been observed in case reports of intracranial meningiomas"? The keywords, "metastatic meningioma," "neck," and "cervical" were used to search articles in English from PubMed, Google Scholar, and Cochrane databases. Articles were selected for full manuscript review after screening for relevance based on title and abstract by two authors (H.C.B.N. and L.J.M.).

Studies were included if the following criteria were met: (1) meningioma and (2) metastatic to the cervical lymph nodes and (3) full-text articles were accessible in English or translatable to English. Abstracts, reviews, reports of animal studies, and cases of metastatic meningioma to regions outside the neck were excluded. Data were extracted from selected studies using the following variables: study author(s), year, age, sex, primary site of tumor, metastatic site of tumor, WHO grade (if available), history of recurrence, treatment modality, and survival at scheduled follow-up.

\section{Case Report}

A 47-year-old female with a history of multiply recurrent orbital meningioma presented with trismus, pain with mastication, swelling along her left zygomatic and temporal region, and worsening diplopia with decreased visual acuity of the left eye. She initially underwent resection of a left-sided skull base meningioma in 2005 (pterional craniotomy approach), with re-resection in 2009 (orbitozygomatic approach) and proton therapy in 2010 for recurrence. Since then, she was followed closely with serial imaging. Her most recent MRI at presentation showed progression of residual disease with a $25.4 \mathrm{~mm} \times 2.0 \mathrm{~mm}$ enhancing mass along the left orbit (Fig. 2a) and a $34.1 \mathrm{~mm} \times 33.1 \mathrm{~mm}$ mass in the masticator space (Fig. 2b). Although fine-needle aspirate of the left infratemporal fossa mass was unrevealing, the decision was made to proceed with surgical resection after multidisciplinary tumor board review. Definitive treatment was undertaken with a transparotid infratemporal fossa resection, orbital exenteration, and anterolateral thigh free flap reconstruction (Fig. 3a-d). Composite left mandibulectomy was also performed and sent for pathology (Fig. 3e). A pathologic appearing level IIA lymph node, which was noticed to be firm and mildly enlarged, was removed during neck exploration for vessels (Fig. 3f). Her postoperative course was uncomplicated. Postoperative MRI demonstrated interval decrease in bulk of the mass centered around the coronoid process of left ascending ramus (Fig. 4a-b). Final pathology showed invasive low-grade meningioma with low Ki-67 index of the main specimen of the bone (Fig. 5a, b), skeletal muscle (Fig. 5c), as well as excised lymph node (Fig. 5d), supporting the rare diagnosis of metastatic meningioma to the cervical lymph node. The patient remained well without no evidence of cancer recurrence at 3- and 6-month follow-up appointments.

\section{Results}

There were 174 nonredundant titles identified from initial screening of databases as described in the methods. Of these, 74 were excluded due to lack of relevance, unavailability in English, systematic reviews, or animal studies. Of the remaining 100 articles, 33 did not have full text available, and as a result, a total 67 articles were identified for review of inclusion in the studies. Of those, 58 were excluded from full manuscript review based on article title and abstract on the basis of lacking involvement 

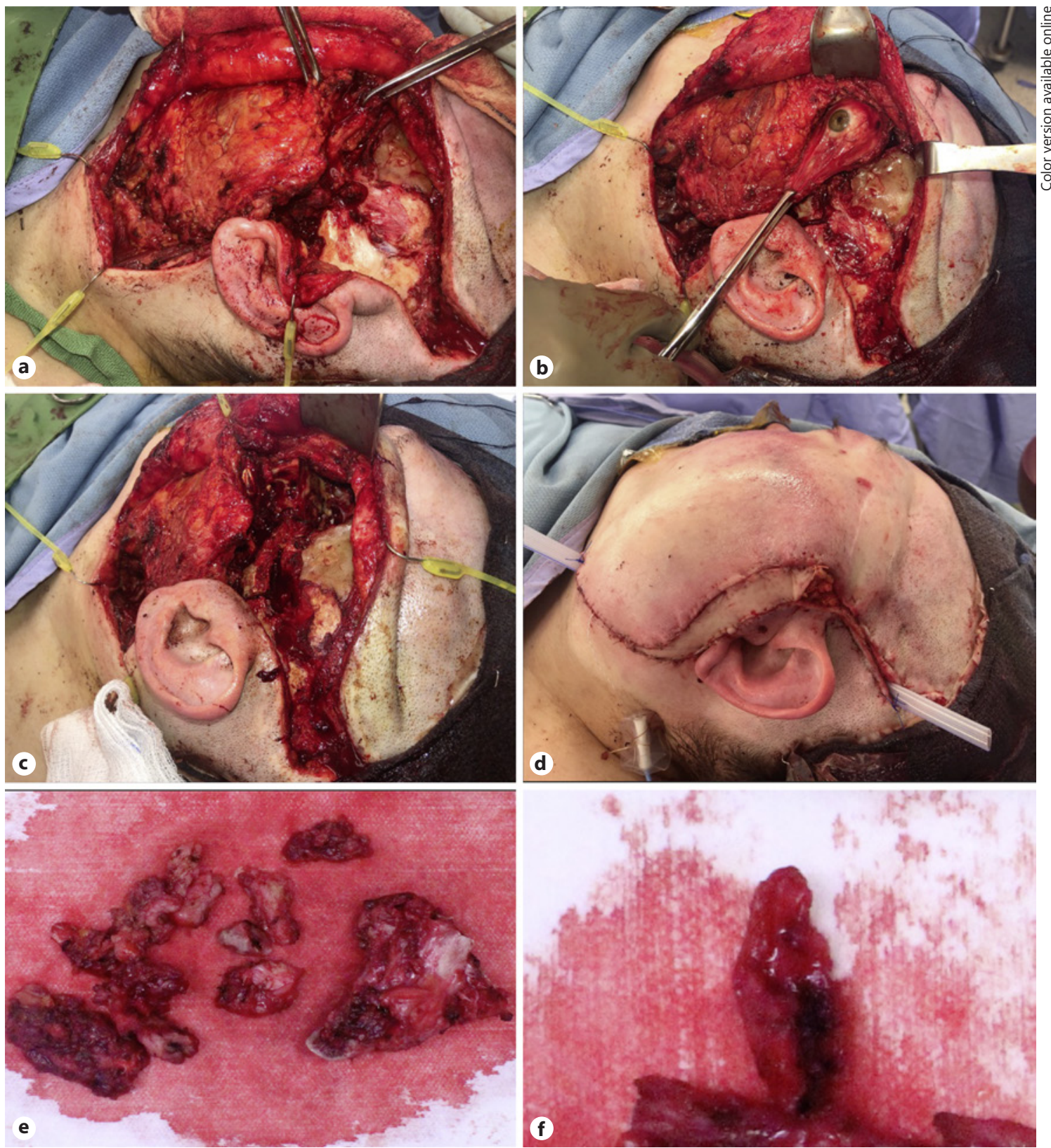

Fig. 3. Transparotid infratemporal fossa resection with ALT free flap reconstruction. a Left composite mandibulectomy involving the posterior mandibular body, ramus, condyle, and coronoid with excision of the glenoid fossa and zygoma. $\mathbf{b}$ Orbital exenteration on the left for the orbital recurrence of tumor after excision of the masseteric and infratemporal fossa tumor. c Revision orbito-zygomatic approach and excision of the middle cranial fossa and orbital apex portions of the tumor. $\mathbf{d}$ Closure with ALT microvascular musculocutaneous free flap and reconstruction of skull base defect. e, f Gross pathology specimen for composite left mandibulectomy and level IIa lymph node, respectively. ALT, anterolateral thigh.

of neck metastasis or wrong subtype of primary tumors. The remaining articles were fully reviewed by two of the authors (H.C.B.N. and L.J.M.), and consensus for inclusion was agreed independently for 9 articles. A list and characterization of the reported cases can be found in Table 1.

Of the 9 cases, 8 were presented as case reports while 1 was presented as a case series [17-24]. All cases were 
Fig.4. Axial contrast-enhanced T1-weighted MRI images of the head postoperatively at the level of the orbit (a) and zygomatic arch (b) showing sequelae of resection of left orbital mass with left orbital exenteration and free flap reconstruction.
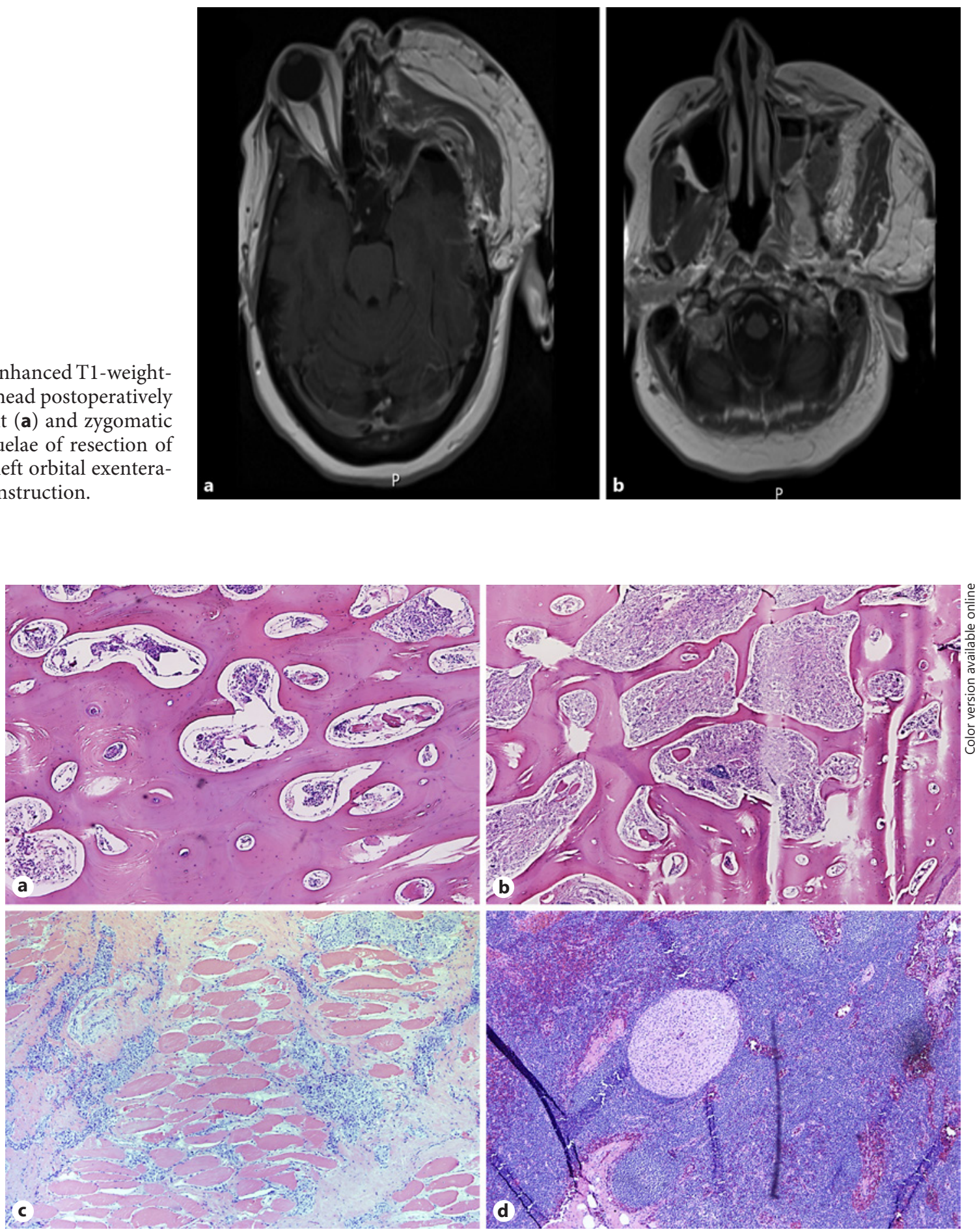

Fig. 5. Hematoxylin-eosin stain showing fusiform cells with oval nuclei, moderate polymorphism, and large atypical nucleolated cells in whorled, concentric arrangement in both the main specimen: bone and bone marrow spaces (a, b), skeletal muscle (c), and the excised cervical lymph node (d). 


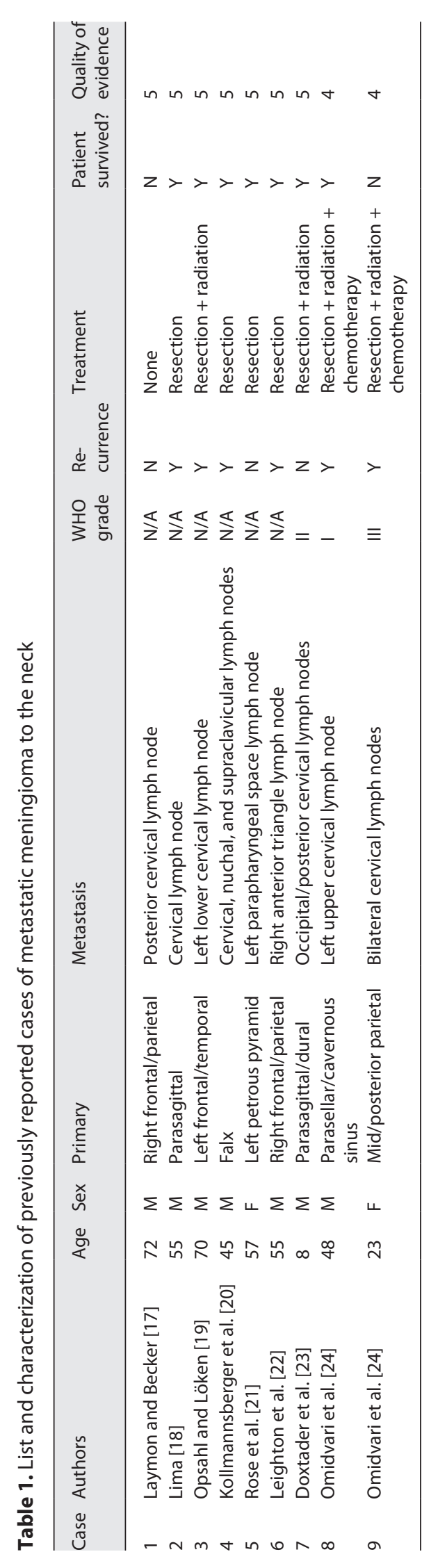

examined based on age, gender, primary site of tumor, metastatic site of tumor, WHO grade (if available), whether the tumor was recurrent, treatment, and survival at follow-up. All cases presented with a primary intracranial meningioma and an enlarging neck mass with subsequent histopathological findings of metastatic meningioma to the cervical lymph nodes. There were no predefined time period constraints in our search, but the resulting cases were from the period of 1949 to 2021. Cases were reported from the USA, France, Germany, and Iran. Most cases were in the adult population aged $23-72$ years, except for 1 case of an 8-year-old patient [23]. There were no differences in sex related to presentation of metastatic cervical disease.

\section{Discussion}

Metastatic meningioma is classically believed to derive from malignant WHO grade III tumors. Here, we report a case of metastasis to the neck from a histologically benign, multiply recurrent orbital meningioma. Although not as common as metastasis to the lungs and liver, cervical spread of intracranial meningiomas has been reported in the literature as far back as 1949. Laymon and Becker [17] reported the first known case of massive right frontal and parietal meningioma with concurrent posterior cervical lymphadenopathy that shared similar pathological findings of an aggressive meningioma. Unfortunately, due to surgical limitations at the time, the patient did not receive any intervention, resulting in mortality. Since then, there have been 8 other case reports of meningiomas with similar disease patterns. The majority of these patients presented with recurrent meningiomas who underwent extensive surgical resection, with or without adjuvant radiation, similar to our patient.

Although the WHO classifications did not exist until the 21st century, pathological reports of these tumors indicate aggressive features such as hypercellularity, smallcell morphology, high mitotic rate, and necrosis, consistent with currently described grade II or III [18-22]. The only known report of WHO grade I meningioma with cervical lymph node metastasis was reported by Omidvari [24]. Here, a 48-year-old male presented with a recurrent meningioma of the parasellar/cavernous sinus and left neck mass status after resection and radiation 9 years before [24]. Cervical metastatic meningioma was also reported in most cases where the patient also received prior surgery, suggesting a possible correlation between cervical spread and surgical resection of primary tumor. 
The mechanism of metastatic spread to the neck remains uncertain. It is hypothesized that violation of the blood-brain barrier during intracranial resection may provide a route of egress for the tumor to access lymphatic channels within the overlying dura and scalp. As such, seeding of tumor cells following surgical resection may account for the high frequency of neck metastasis observed in reported cases [20, 22, 23]. It is also worth noting that in all cases, metastasis was not detected elsewhere. Fortunately, almost all patients that received at least standard treatment, including surgery, survived on scheduled follow-up. Mortality was observed in one case of a 23-year-old female patient with primary grade III anaplastic meningioma who also received systemic interferon alpha2-b and external beam radiotherapy with a total dose of 50 Gy to the entire cervical lymph nodes [24]. She succumbed to disease 5 months following neck recurrence due to rapidly progressive lymphadenopathy. These findings suggest that WHO grade classification may be a better indication of prognosis than tumor spread. As presented here, local therapy, including surgery and stereotactic radiosurgery, can be extremely effective in cases of restricted metastasis or oligometastatic disease. We also acknowledge that there are limitations to our study as it only pertains to previous case reports of metastatic meningiomas to the cervical region, which are both uncommon and less reliable as a source of evidence.

The 21 st century also bore witness to unprecedented growth in genomic technology, with the advent of nextgeneration high-throughput sequencing that opened doors to rapid molecular profiling of many diseases, including the characterization and identification of new and recurring genetic and epigenetic alterations of meningiomas. In fact, in 2016, the WHO classification incorporated molecular characteristics as part of many brain tumors including meningiomas [25]. Here, we highlight the most recent progresses toward not only understanding the molecular underpinnings of meningioma but also developing more effective diagnostic and treatment options.

Mutations in the tumor suppressor gene NF2 were the first to be identified as a genetic cause of meningiomas. In fact, a diagnosis of meningioma in the pediatric population often prompted further genetic testing for NF2 mutations $[6,8]$. Since then, numerous other genes have been implicated, including TRAF7, AKT1, KLF4, SMO, PIK3CA, DAL-1, TIMP1, TIMP3, FOXM1, GLI transcription genes, MEG3, SMARCE1, STA T3, PTCH1, and TP73 [15, 26-39]. Interestingly, disease progression and malignancy are more often associated with chromosomal aberra- tions, rather than point mutations. Loss of chromosomes $1 \mathrm{p}, 6 \mathrm{q}, 10 \mathrm{p}, 10 \mathrm{q}, 14 \mathrm{q}$, and $18 \mathrm{q}$ are most commonly reported in atypical and anaplastic meningiomas [30-33]. Especially, losses of $1 \mathrm{p}$ and $14 \mathrm{q}$ were reportedly associated with worsened prognosis [34-36].

Changes in the epigenetic landscapes have also been shown to be of equal, if not more, importance in tumorigenesis of meningiomas [40]. Specifically, dysregulation in DNA methylation of the promoter regions of IGF2BP1, PENK, RASSF1A, HOXA6, HOXA9, TP53, WINK2, and $H I F-3 \alpha 4$ is associated with both progression and malignant transformation [38-41]. Chromatin instability was also reported in association with meningiomas, with altered expression levels of several regulators of chromatin architecture such as KDM5C, KDM6A, and SMARCB1 [37].

Perhaps even more exciting is the advent of miRNA and their regulatory role in cancer biology. MicroRNAs (miRNAs) refer to a class of small endogenous noncoding RNAs that regulate gene expression at the post-transcriptional level $[42,43]$. Emerging studies have shown that miRNAs play critical roles in tumorigenesis and cancer progression. High-throughput sequencing has enabled the characterization of hundreds of differentially expressed miRNAs in meningioma versus healthy meninges, as well as high-grade versus low-grade meningiomas. For example, $m i R-21$ has been overexpressed in several tumors and may be a promising clinical biomarker for possible therapeutic targeting $[44,45]$. In atypical and anaplastic tumors, significant reductions in $m i R-145$ have been shown in comparison with benign meningiomas [46]. Low miR-224 expression has exhibited significantly prolonged overall and recurrence-free survival [47]. Other microRNAs associated with recurrence and higher grade meningiomas include $m i R-34 a, m i R 218$, $m i R-219$ $5 p$, and $m i R-335$; however, these targets need further independent validations $[42,43]$. Multiple studies have suggested that circulatory miRNAs bear similar expression pattern as those in the CSF, and thus hold promising potentials in being used as biomarkers for noninvasive liquid biopsy [48-50].

\section{Conclusion}

Metastatic meningioma to the cervical region is an extremely rare but important differential diagnosis to consider in patients presenting with a neck mass, especially in those with a history of intracranial tumors treated with local resection. Meningiomas of any stage can metastasize 
to this region, but a higher grade tumor can indicate worse prognosis. Surgery is an effective treatment for recurrent meningioma even though it can induce cervical spread of the disease. Finally, new insights into the multiple genetic and epigenetic abnormalities of meningiomas open doors for novel diagnostic and therapeutic options.

\section{Statement of Ethics}

An ethics statement is not applicable because this study is based exclusively on the published literature. Written informed consent was obtained from the patient for publication of this case report and any accompanying images.

\section{Conflict of Interest Statement}

The authors have no funding, financial relationships, or conflicts of interest to disclose.

\section{Funding Sources}

This manuscript did not receive any funding.

\section{Author Contributions}

H.C.B.N. and L.J.M. performed the systematic reviews. H.C.B.N., L.J.M., and R.M.B. wrote the manuscript. H.C.B.N., L.J.M., K.P., I.H.C., A.A.C., and R.M.B. were involved in the surgical operation. S.A. and K.C. performed pathological reports.

\section{Data Availability Statement}

All data generated or analyzed during this study are included in this article. Further enquiries can be directed to the corresponding author.

\section{References}

1 Catala M. Embryonic and fetal development of structures associated with the cerebro-spinal fluid in man and other species. Part I: the ventricular system, meninges and choroid plexuses. Arch Anat Cytol Pathol. 1998 Jan 1; 46(3):153-69.

2 Marosi C, Hassler M, Roessler K, Reni M, Sant M, Mazza E, et al. Meningioma. Crit Rev Oncol Hematol. 2008 Aug 1;67(2):153-71.

3 Ostrom QT, Gittleman H, Fulop J, Liu M, Blanda R, Kromer C, et al. CBTRUS statistical report: primary brain and central nervous system tumors diagnosed in the United States in 2008-2012. Neuro Oncol. 2015 Oct 1;17 Suppl 4(Suppl 4):iv1-62.

4 Louis DN, Ohgaki H, Wiestler OD, Cavenee WK, Burger PC, Jouvet A, et al. The 2007 WHO classification of tumours of the central nervous system. Acta Neuropathol. 2007 Aug; 114(2):97-109.

5 Klaeboe L, Lonn S, Scheie D, Auvinen A, Christensen HC, Feychting M, et al. Incidence of intracranial meningiomas in Denmark, Finland, Norway and Sweden, 1968-1997. Int J Cancer. 2005 Dec 20;117(6):996-1001.

6 Evans DG, Salvador H, Chang VY, Erez A, Voss SD, Schneider KW, et al. Cancer and central nervous system tumor surveillance in pediatric neurofibromatosis 1 . Clin Cancer Res. 2017 Jun 15;23(12):e46-53.

7 Tufan K, Dogulu F, Kurt G, Emmez H, Ceviker N, Baykaner MK. Intracranial meningiomas of childhood and adolescence. Pediatr Neurosurg. 2005;41(1):1-7.

8 Perry A, Giannini C, Raghavan R, Scheithauer BW, Banerjee R, Margraf L, et al. Aggressive phenotypic and genotypic features in pediatric and NF2-associated meningiomas: a clinicopathologic study of 53 cases. J Neuropathol Exp Neurol. 2001 Oct 1;60(10):9941003.

9 Whittle IR, Smith C, Navoo P, Collie D. Meningiomas. Lancet. 2004 May 8;363(9420): 1535-43.

10 Ostrom QT, Gittleman H, de Blank PM, Finlay JL, Gurney JG, McKean-Cowdin R, et al. American brain tumor association adolescent and young adult primary brain and central nervous system tumors diagnosed in the United States in 2008-2012. Neuro Oncol. 2016 Jan 1;18 Suppl 1(Suppl 1):11-50.

11 Simpson D. The recurrence of intracranial meningiomas after surgical treatment. J Neurol Neurosurg Psychiatry. 1957 Feb;20(1):22.

12 Figueroa BE, Quint DJ, McKeever PE, Chandler WF. Extracranial metastatic meningioma. Br J Radiol. 1999 May;72(857):513-6.

13 Pramesh CS, Saklani AP, Pantvaidya GH, Heroor AA, Naresh KN, Sharma S, et al. Benign metastasizing meningioma. Jpn J Clin Oncol. 2003 Feb 1;33(2):86-8.

14 Sioka C, Kyritsis AP. Chemotherapy, hormonal therapy, and immunotherapy for recurrent meningiomas. J Neurooncol. 2009 Mar;92(1):1-6.

15 Preusser M, Brastianos PK, Mawrin C. Advances in meningioma genetics: novel therapeutic opportunities. Nat Rev Neurol. 2018 Feb;14(2):106-15.

16 Moher D, Liberati A, Tetzlaff J, Altman DG. Preferred reporting items for systematic reviews and meta-analyses: the PRISMA statement. Int J Surg. 2010 Jan 1;8(5):336-41.

17 Laymon CW, Becker FT. Massive metastasizing meningioma involving the scalp. Arch Derm Syphilol. 1949 Jun 1;59(6):626-35.
18 Lima A. Metastase cervical de um meningioma parassagital. Rev Esp Otoneurooftalmol. 1951;10:313-6.

19 Opsahl JRR, Loken AC. Meningioma with metastases to cervical lymph nodes: case report. Acta Path et Microbiol Scandinav. 1965 Sep;64(3):294-8.

20 Kollmannsberger A, Kazner E, Prechtel K, Stochdorph O. Extracranial metastasis of meningeal tumors. Malignant meningioma with regional lymph node metastasis. Zentralbl Neurochir. 1975 Jan 1;36(1):27-36.

21 Rose WS, Makhija MC, Sattenspiel S. Meningioma presenting as a tumor in the neck. AJR Am J Roentgenol. 1980 May 1;134(5):1070-2.

22 Leighton SE, Rees GL, McDonald B, AlunJones T. Metastatic meningioma in the neck. J Laryngol Otol. 1991 Mar;105(3):229-31.

23 Doxtader EE, Butts SC, Holsapple JW, Fuller CE. Aggressive pediatric meningioma with soft tissue and lymph node metastases: a case report. Pediatr Dev Pathol. 2009 May;12(3): 244-8.

24 Omidvari S, Nasrolahi H, Daneshbod Y, Bagheri N, Negahban S, Mohammadianpanah $\mathrm{M}$, et al. Cervical lymph node metastases from meningioma: report of two cases and treatment outcome. Middle East J Cancer. 2010; 1(1):45-9.

25 Louis DN, Perry A, Reifenberger G, Von Deimling A, Figarella-Branger D, Cavenee WK, et al. The 2016 World Health Organization classification of tumors of the central nervous system: a summary. Acta Neuropathol. 2016 Jun;131(6):803-20.

26 Yuzawa S, Nishihara H, Tanaka S. Genetic landscape of meningioma. Brain Tumor Pathol. 2016 Oct;33(4):237-47.

Nguyen/Mady/Panara/Andrianus/ Cooper/Chen/Chalian/Brody 
27 Fontaine B, Rouleau GA, Seizinger BR, Menon AG, Jewell AF, Martuza RL, et al. Molecular genetics of neurofibromatosis 2 and related tumors (acoustic neuroma and meningioma). Ann N Y Acad Sci. 1991 Jan 1;615: 338-43.

28 Mawrin C, Perry A. Pathological classification and molecular genetics of meningiomas. J Neurooncol. 2010 Sep;99(3):379-91.

29 Clark VE, Erson-Omay EZ, Serin A, Yin J, Cotney J, Özduman K, et al. Genomic analysis of non-NF2 meningiomas reveals mutations in TRAF7, KLF4, AKT1, and SMO. Science. 2013 Mar 1;339(6123):1077-80.

30 Bello MJ, Pestaña A, Rey JA, De Campos JM, Kusak ME, Vaquero J, et al. Allelic loss at $1 \mathrm{p}$ is associated with tumor progression of meningiomas. Genes Chromosomes Cancer. 1994 Apr;9(4):296-8.

31 Simon M, von Deimling A, Larson JJ, Wellenreuther R, Kaskel P, Waha A, et al. Allelic losses on chromosomes 14, 10, and 1 in atypical and malignant meningiomas: a genetic model of meningioma progression. Cancer Res. 1995 Oct 15;55(20):4696-701.

32 Weber RG, Boström J, Wolter M, Baudis M, Collins VP, Reifenberger G, et al. Analysis of genomic alterations in benign, atypical, and anaplastic meningiomas: toward a genetic model of meningioma progression. Proc Natl Acad Sci U S A. 1997 Dec 23;94(26):1471924.

33 Aizer AA, Abedalthagafi M, Linda Bi W, Horvath $\mathrm{MC}$, Arvold ND, Al-Mefty O, et al. A prognostic cytogenetic scoring system to guide the adjuvant management of patients with atypical meningioma. Neuro Oncol. 2015 Aug 30;18(2):269-74.

34 Sulman EP, Dumanski JP, White PS, Zhao H, Maris JM, Mathiesen T, et al. Identification of a consistent region of allelic loss on 1p32 in meningiomas: correlation with increased morbidity. Cancer Res. 1998 Aug 1;58(15): 3226-30.

35 Tabernero MD, Espinosa AB, Maíllo A, Sayagués JM, Alguero MD, Lumbreras E, et al. Characterization of chromosome 14 abnormalities by interphase in situ hybridization and comparative genomic hybridization in 124 meningiomas: correlation with clinical, histopathologic, and prognostic features. Am J Clin Pathol. 2005 May 1;123(5):744-51.

36 Linsler S, Kraemer D, Driess C, Oertel J, Kammers K, Rahnenführer J, et al. Molecular biological determinations of meningioma progression and recurrence. PLoS One. $2014 \mathrm{Apr}$ 10;9(4):e94987.

37 Galani V, Lampri E, Varouktsi A, Alexiou G, Mitselou A, Kyritsis AP. Genetic and epigenetic alterations in meningiomas. Clin Neurol Neurosurg. 2017 Jul 1;158:119-25.

38 Zhang Y, Xiong Y, Yarbrough WG. ARF promotes MDM2 degradation and stabilizes p53: ARF-INK4a locus deletion impairs both the $\mathrm{Rb}$ and $\mathrm{p} 53$ tumor suppression pathways. Cell. 1998 Mar 20;92(6):725-34.

39 Yin D, Xie D, Hofmann WK, Miller CW, Black KL, Koeffler HP. Methylation, expression, and mutation analysis of the cell cycle control genes in human brain tumors. Oncogene. 2002 Nov;21(54):8372-8.

40 McTavish N, Copeland LA, Saville MK, Perkins ND, Spruce BA. Proenkephalin assists stress-activated apoptosis through transcriptional repression of NF-kappaB- and p53-regulated gene targets. Cell Death Differ. 2007 Sep;14(9):1700-10.

41 Kishida Y, Natsume A, Kondo Y, Takeuchi I, An B, Okamoto Y, et al. Epigenetic subclassification of meningiomas based on genomewide DNA methylation analyses. Carcinogenesis. 2012 Feb 1;33(2):436-41.
42 El-Gewely MR, Andreassen M, Walquist M, Ursvik A, Knutsen E, Nystad M, et al. Differentially expressed microRNAs in meningiomas grades I and II suggest shared biomarkers with malignant tumors. Cancers. 2016 Mar; $8(3): 31$.

43 Wang L, Chen S, Liu Y, Zhang H, Ren N, Ma $\mathrm{R}$, et al. The biological and diagnostic roles of MicroRNAs in meningiomas. Rev Neurosci. 2020 Oct 1;31(7):771-8.

44 Wijnhoven BP, Michael MZ, Watson DI. MicroRNAs and cancer. Br J Surg. 2007 Jan; 94(1):23-30.

45 TraNgas T. Expression of stem cell marker nestin and microRNA-21 in meningiomas. Turk Neurosurg. 2015;25(4):574-7.

46 Kliese N, Gobrecht P, Pachow D, Andrae N, Wilisch-Neumann A, Kirches E, et al. miRNA-145 is downregulated in atypical and anaplastic meningiomas and negatively regulates motility and proliferation of meningioma cells. Oncogene. 2013 Sep;32(39):4712-20.

47 Wang M, Deng X, Ying Q, Jin T, Li M, Liang C. MicroRNA-224 targets ERG2 and contributes to malignant progressions of meningioma. Biochem Biophys Res Commun. 2015 May 1;460(2):354-61.

48 Shalaby T, Grotzer MA. Tumor-associated CSF microRNAs for the prediction and evaluation of CNS malignancies. Int J Mol Sci. 2015 Dec;16(12):29103-19.

49 Abedalthagafi M, Bi WL, Aizer AA, Merrill $\mathrm{PH}$, Brewster R, Agarwalla PK, et al. Oncogenic PI3K mutations are as common as AKT1 and SMO mutations in meningioma. Neuro Oncol. 2016 May 1;18(5):649-55.

50 Yuzawa S, Nishihara H, Yamaguchi S, Mohri $\mathrm{H}$, Wang L, Kimura T, et al. Clinical impact of targeted amplicon sequencing for meningio$\mathrm{ma}$ as a practical clinical-sequencing system. Mod Pathol. 2016 Jul;29(7):708-16. 